\title{
Attendance Management System using Face-Recognition
}

\author{
Mrunal Aware, Prasad Labade, Manish Tambe, Aniket Jagtap, Chinmay Beldar
}

Department of Computer Engineering, MIT Polytechnic, Pune, Maharashtra, India

\begin{abstract}
Article Info

Volume 7, Issue 3

Page Number: 336-341

Publication Issue :

May-June-2021

\section{Article History}

Accepted : 15 May 2021

Published : 22 May 2021

Nowadays Educational institutions are concerned about regularity of student attendance. Even in pandemic situation attendance is still a major issue in schools and colleges. Mainly there are two conventional methods of marking attendance which are calling out the roll call or by taking student sign on paper. They both were more time consuming and difficult. Hence, there is a requirement of computer-based student attendance management system which will assist the faculty for maintaining attendance record automatically. In this project we have implemented the automated attendance system using 'TKINTER' and 'PYTHON'. We have projected our ideas to implement an "Automated Attendance System Based on Face Recognition". The application includes face identification, which saves time as well as being purely softwere based it can be flagged as eco-friendly as it reduces the use of paper. This system also eliminates the chances of fake attendance because of the face being used as a biometric for authentication. Hence, this system can be implemented in a field where attendance plays an important role. The proposed system is designed in TKINTER platform supported with a script of PYTHON as well as SQL database. The algorithm used in the system is based on image comparison on the basis of the encoded values of the face from the image from database with the image recorded by the system in run time. The system has output in the form of excel sheet.
\end{abstract}

Keywords : Attendance system, Python, Machine Learning, Tkinter, MySQL.

\section{INTRODUCTION}

The Attendance System using Face - Recognition is a replacement way method for the traditional way of marking attendance. The proposed system is python , tkinter based system supported with MySQL database. This system can be implemented on a single faculty system of a particular institute. This system is proposed to be based on biometrics i.e. Face
Authentication. Since there is presence of biometrics, this system completely eliminates the chances of fake attendance which is a problem with the traditional methods of attendance.

The Attendance management is the significant process that were carry out in every institute to monitor the performance of the student. Every institute does this is its own way. Some of there

Copyright: @ the author(s), publisher and licensee Technoscience Academy. This is an open-access article distributed under the terms of the Creative Commons Attribution Non-Commercial License, which permits unrestricted non-commercial use, distribution, and reproduction in any medium, provided the original work is properly cited 
institute use the old paper or file-based system and some have adopted strategies of automated attendance system using some biometric technique. A facial recognition system is a computerized software which is suited for determining or validating a person by performing comparisons on patterns based on their facial appearances. In this system OpenCV \& Face Recognition libraries were used which are one of the popular libraries for face detection by using these libraries system first capturing the student photos and storing them into the database which were further used for the training purpose after that at the time of attendance when system camera get on system will detect the faces that were present in the frame the faces were detected by using HOG i.e. (Histogram of Oriented Gradients) which were carrier out in the system. after that if image that were present in the frame is tilted then Face Landmark Estimation algorithm will be carried out and face will be transformed to be as close as possible to perfectly centered. After that system will encode all the images that were present in the database as well as the face which were detected in the frame. For performing encoding Deep Conversional Neural Network algorithm will get carried out \& for each face 128 measurements were generated then the measurements of the face that were detected in frame it get compared with the measurements of the faces that were present in the image which is earlier stored in the database. So at last by using simple liner SVM algorithm system will find the person in database of know peoples (i.e. capture at the starting of the project) who has closest measurements to the image that were detected by camera. After finding perfect match system will generate the name and date \& time \& present mark and store the entry in CSV file. Which were further uploaded on the database and also user can open it with Microsoft Excel.

\section{LITREATURE SURVEY}

Using real time computer vision algorithms in automatic attendance management systems This paper introduces a new approach in automatic attendance management systems, extended with computer vision algorithms. The Proposed system uses real time face detection algorithms integrated on an existing Learning Management System (LMS), which automatically detects and registers students attending on a lecture. The system represents a supplemental tool for instructors, combining algorithms used in machine learning with adaptive methods used to track facial changes during a longer period of time. [1]

Face Recognition-based Lecture Attendance System proposed a system that takes the attendance of students for classroom lecture. The system takes attendance automatically using face recognition. However, it is difficult to estimate the attendance precisely using each result of face recognition independently because the face detection rate is not sufficiently high. In this paper, we propose a method for estimating the attendance precisely using all the results of face recognition obtained by continuous observation.[2]

Automatic Control of students' attendance in Classrooms Using RFID Radio frequency identification (RFID) is one of the automatic identification technologies more in vogue nowadays. There is a wide research and development in this area trying to take maximum advantage of this technology, and in coming years many new applications and research areas will continue to appear.[3]

Face Recognition based Attendance Management System using Machine Learning The most arduous task in any organization is attendance marking. In this paper we proposed an automated attendance management system which tackles the predicament of recognition of faces in biometric systems subject to 
different real time scenarios such as illumination, rotation and scaling. [4]

\section{METHODOLOGY}

The Attendance management is the significant process that were carry out in every institute to monitor the performance of the student. Every institute does this is its own way. Some of there institute use the old paper or file-based system and some have adopted strategies of automated attendance system using some biometric technique.

A facial recognition system is a computerized software which is suited for determining or validating a person by performing comparisons on patterns based on their facial appearances. In this system OpenCV \& Face Recognition libraries were used which are one of the popular libraries for face detection by using these libraries system first capturing the student photos and storing them into the database which were further used for the training purpose after that at the time of attendance when system camera get on system will detect the faces that were present in the frame the faces were detected by using HOG i.e. (Histogram of Oriented Gradients) which were carrier out in the system. after that if image that were present in the frame is tilted then Face Landmark Estimation algorithm will be carried out and face will be transformed to be as close as possible to perfectly cantered.

After that system will encode all the images that were present in the database as well as the face which were detected in the frame. For performing encoding Deep Conversional Neural Network algorithm will get carried out \& for each face 128 measurements were generated then the measurements of the face that were detected in frame it get compared with the measurements of the faces that were present in the image which is earlier stored in the database.
So at last by using simple liner SVM algorithm system will find the person in database of know peoples (i.e. capture at the starting of the project) who has closest measurements to the image that were detected by camera. After finding perfect match system will generate the name and date \& time \& present mark and store the entry in CSV file. Which were further uploaded on the database and also user can open it with Microsoft Excel.

\section{PROPOSED SYSTEM}

\section{Image acquisition:}

Image is acquire using a high definition camera which is placed in the classroom or lab. This image is given as an input to the system. All title and author details must be in single-column format and must be centered.

\section{Dataset Creation:}

Dataset of student is created before the recognition process. Dataset was created only to train this system we are going to create a dataset of the whole class which involve their name, roll number department and images of the student in different variations. Whenever we register student's data and image in our system to create dataset, deep learning applies to each face to compute 128-d facial features and store in student face data file to recall that face in recognition process. This process is applying to each image taken during registration.

\section{Face Detection and Extraction:}

Face detection is important as the image taken through the camera given to the system, face detection algorithm applies to identify the human faces in that image, the number of image processing algorithms are introduced to detect faces in an image 
and also the location of that detected faces. We have used HOG method to detect human faces in given image.

\section{Face Positioning:}

There are 68 specific points in a human face. In other words, we can say 68 face landmarks. The main function of this step is to detect landmarks of faces and to position the image. A python script is used to automatically detect the face landmarks and to position the face as much as possible without distorting the image.

\section{Face Encoding:}

Once the faces are detected in the given image, the next step is to extract the unique identifying facial feature for each image. Basically, whenever we get localization of face, the 128 key facial point are extracted for each image given input which are highly accurate and these 128- $d$ facial points are stored in data file for face recognition.

\section{Face matching:}

This is last step of face recognition process. We have used the one of the best learning techniques that is deep metric learning which is highly accurate and capable of outputting real value feature vector. The proposed system ratifies the faces, constructing the 128-d embedding (ratification) for each. Internally compare faces function is used to compute the Euclidean distance between face in image and all faces in the dataset. If the current image is matched with the $60 \%$ threshold with the existing dataset, it will move to attendance marking.

\section{Attendance Marking:}

Once the face is identified with the image stored in SQL database, python generate roll numbers of present students and return that, when data is returned, the system generates attendance table which includes the name, roll number, date, day and time with corresponding subject id. And then passes the data to python to store the table into an CSV file automatically. Letter staff can open that file into the excel sheet to edit the sheet and make changes in it.

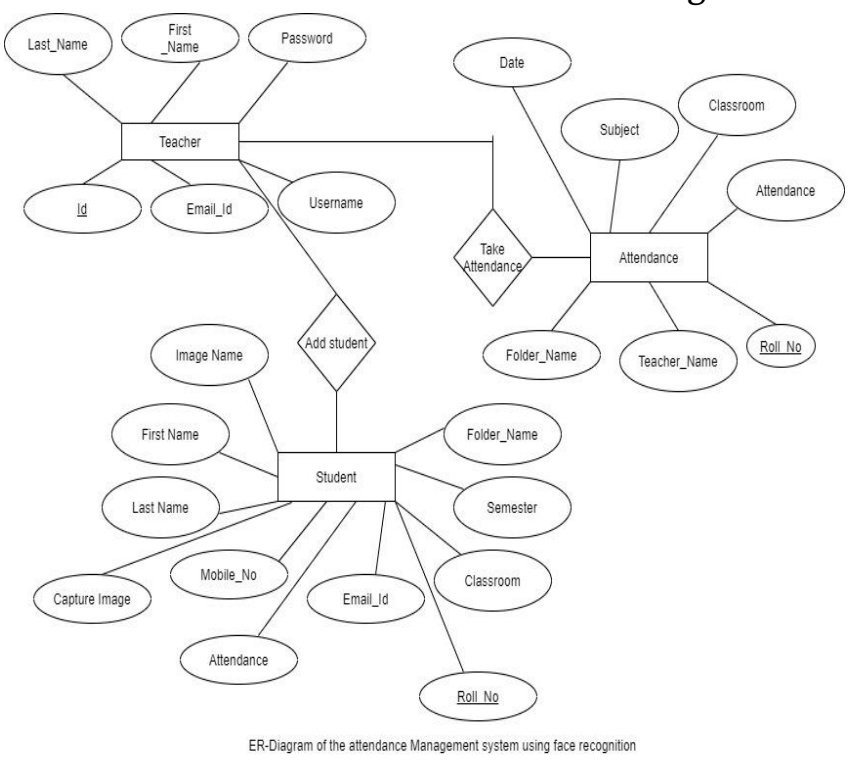

Fig 1. ER-DIAGRAM

Figure 1 shows the Entity-Relation diagram of the proposed system.

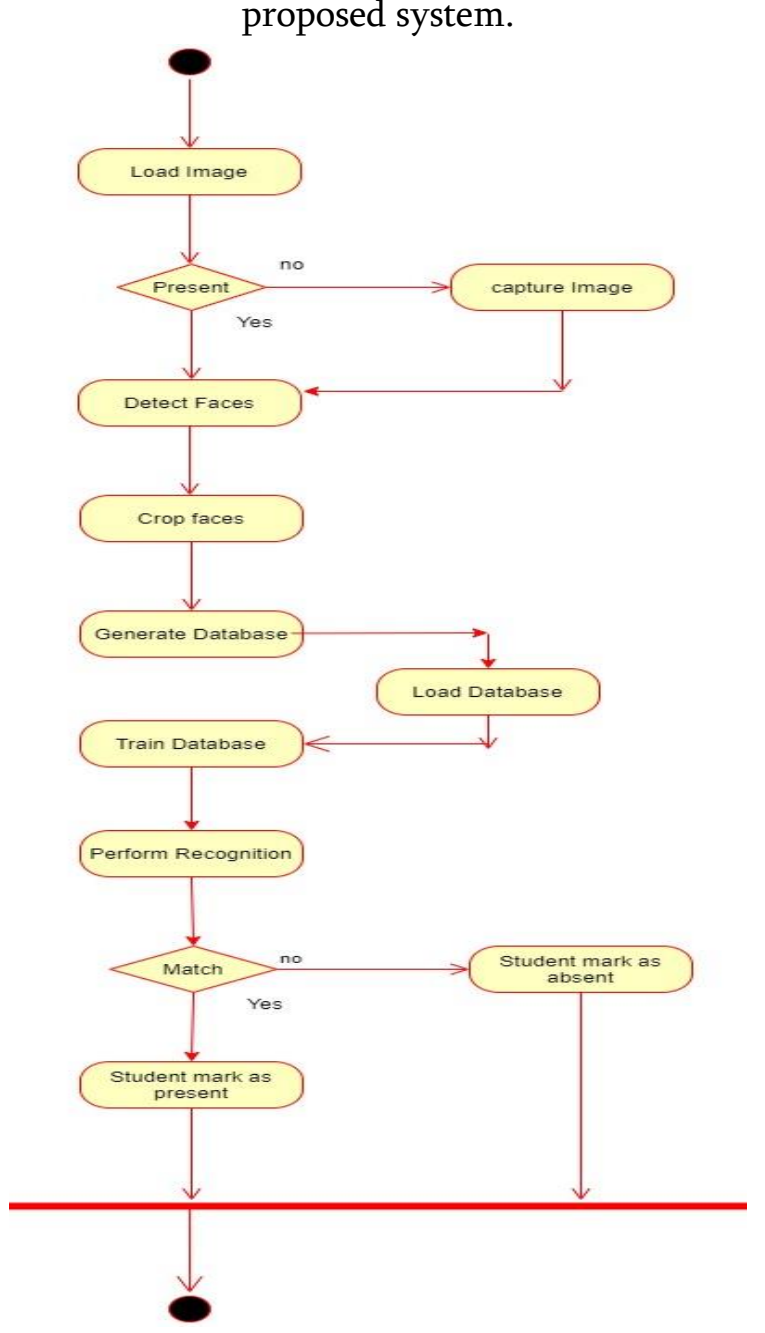


Fig 2. ACTIVITY DIAGRAM

*Figure 2 shows activity diagram of the proposed system

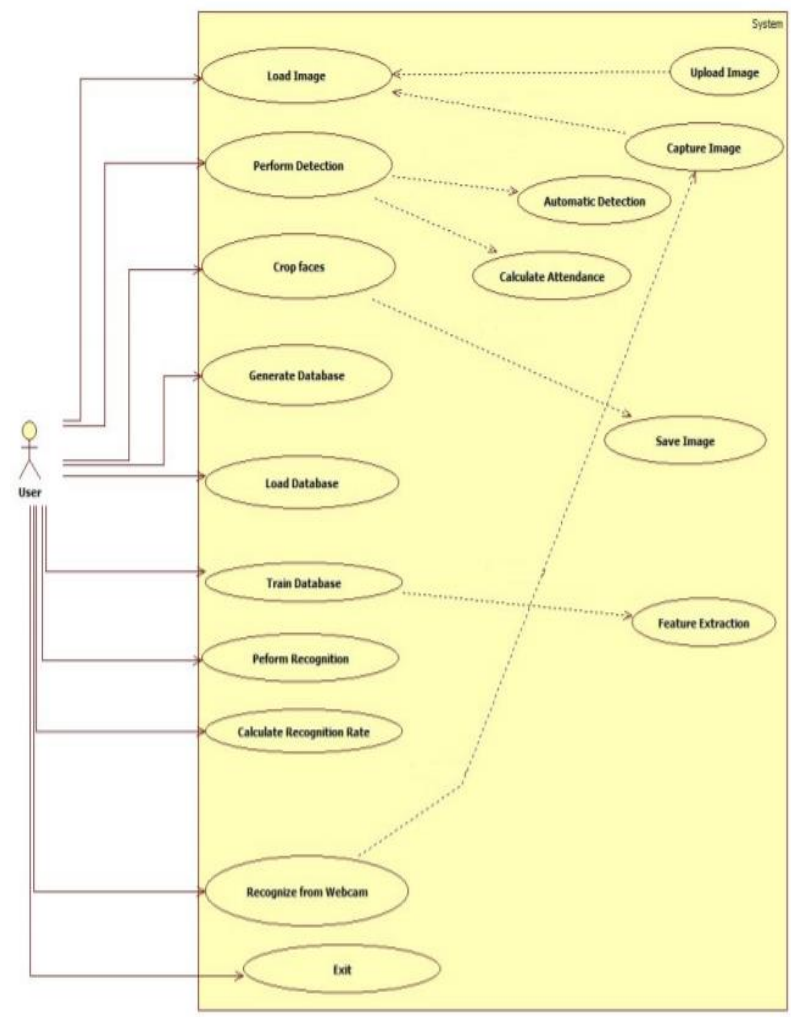

Fig 3. USE-CASE DIAGRAM

*Figure 3 shows the User Interaction diagram of the proposed system.
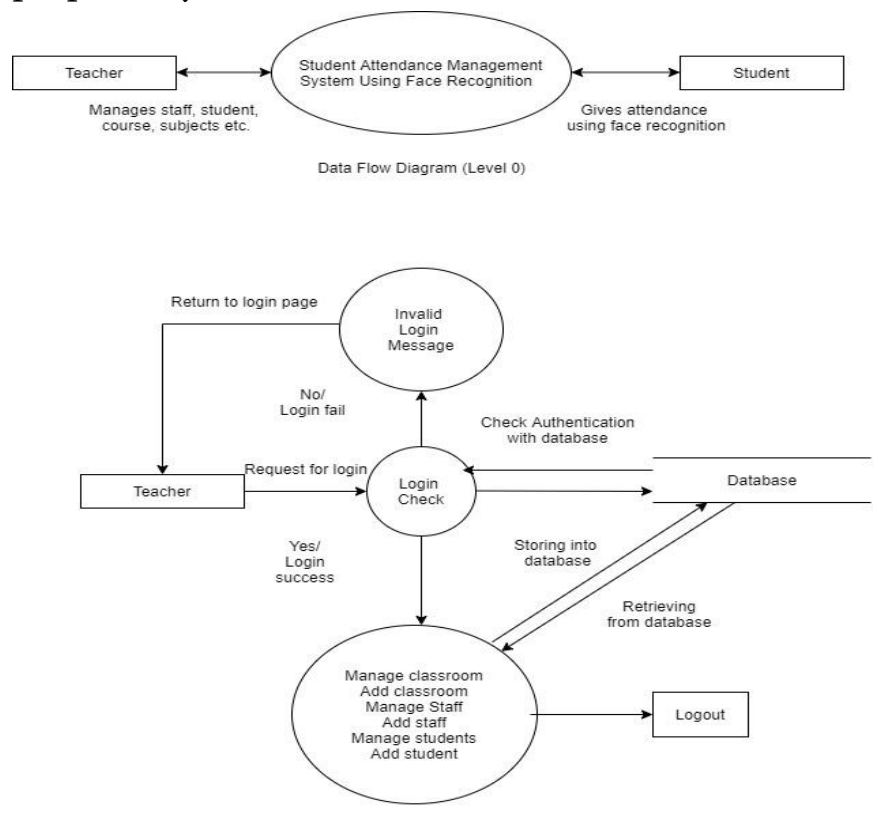

Data Flow Diagram of Admin (Level 1)

Fig 4. DATA FLOW DIAGRAM ( LVL 0 \& LVL 1)

*Figure 4 shows the Data flow diagram on lvl 0 and $\operatorname{lvl} 1$
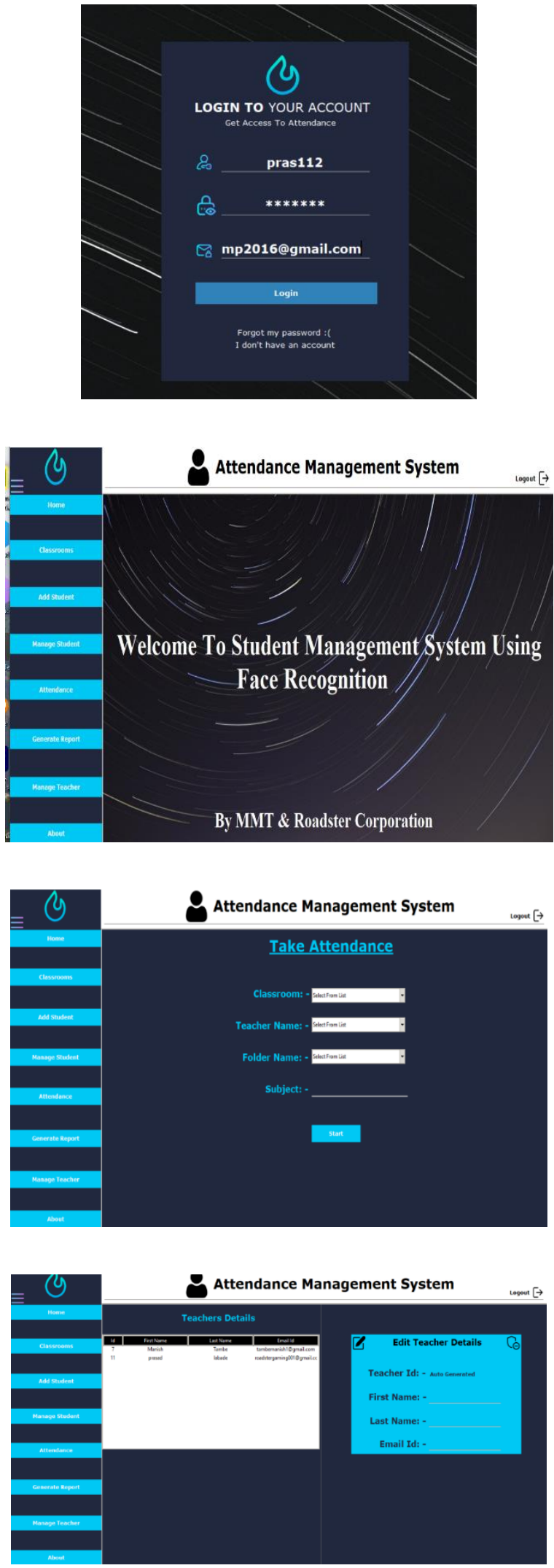

Fig 4 , 5, 6, 7 OUTPUTS

*Figure 4,5,6,7 show the screenshots of the output screen of the system. 


\section{ADVANTAGES OF THE SYSTEM}

The proposed system has a much simpler and efficient algorithm. The system is simpler because of use easy and user-friendly Framework. It has a more efficient algorithm along with much less complex database configurations. The system is more efficient because of being platform independent.

\section{VI.CONCLUSION}

In order to obtain the attendance of individuals and to record the entry and exit, the proposed system can be used. The system can widely be used in the institutions/organizations. The proposed system takes attendance of each student by continuous observation at the entry and exit points. The result of our preliminary experiment shows improved performance in the estimation of the attendance compared to the traditional attendance marking systems.

\section{REFERENCES}

[1]. Dipti Kumbhar , Prof. Dr. Y. S. Angal Department of Electronics and Telecommunication, Smart Attendance System using Computer Vision and Machine Learning yogeshangal@yahoo.co.in IRJET 1/4/2017]

[2]. P. Visalakshi, Sushant Ashish Assistant Professor ,Department of Computer Science and Engineering SRM Institute of Science and Technology, Chennai, Tamil Nadu, ATTENDANCE SYSTEM USING MULTI-FACE RECOGNITION , IEEE 27/5/2016]

[3]. CH. VINOD KUMAR , DR. K. RAJA KUMAR PG Scholar, Dept of CS\& SE, Andhra University, Vishakhapatnam, AP, India. Assistant Professor, Dept of CS\& SE, Andhra University, Vishakhapatnam, AP, India. Face Recognition Based Student Attendance System with OpenCV $15 / 12 / 2014]$
[4]. Face Recognition based Attendance Management System using Machine Learning. IRJET 06, June 2011 ]

[5]. Ashish Choudhary,Abhishek Tripathi,Abhishek Bajaj,Mudit Rathi and B.M Nandini Information Science and Engineering, The National Institute of Engineering, Automatic Attendance System Using Face Recognition. IRJET 12/5/2013]

[6]. Anushka Waingankar1, Akash Upadhyay, Ruchi Shah, Nevil Pooniwala, Prashant Kasambe. Face Recognition based Attendance Management System using Machine Learning IEEE 8/6/2012]

[7]. By Alan D. Moore, Python GUI Programming with Tkinter Develop Responsive and Powerful GUI Applications with Tkinter , IEEE 4/11/2010]

\section{Cite this article as :}

Mrunal Aware, Prasad Labade, Manish Tambe, Aniket Jagtap, Chinmay Beldar, "Attendance Management System using Face-Recognition", International Journal of Scientific Research in Computer Science, Engineering and Information Technology (IJSRCSEIT), ISSN : 2456-3307, Volume 7 Issue 3, pp. 336-341, May-June 2021. Available at doi : https://doi.org/10.32628/CSEIT217370 Journal URL : https://ijsrcseit.com/CSEIT217370 